\title{
Dissociative Identity Disorder
}

National Cancer Institute

\section{Source}

National Cancer Institute. Dissociative Identity Disorder. NCI Thesaurus. Code C94330.

A disorder characterized by the presence of two or more identities with distinct patterns

of perception and personality which recurrently take control of the person's behavior; this is accompanied by a retrospective gap in memory of important personal information that far exceeds ordinary forg etfulness. The changes in identity are not due to substance use or to a general medical condition. 